\title{
Design of an affordable, modular implant device for soft tissue tension assessment and range of motion tracking during total hip arthroplasty
}

This paper was downloaded from TechRxiv (https://www.techrxiv.org).

\section{LICENSE}

CC BY 4.0

SUBMISSION DATE / POSTED DATE

$05-11-2021 / 09-11-2021$

\section{CITATION}

Wei, Jonathan; Blaauw, Bryan; Pol, Dieter GM van der; Saldívar, Mauricio Cruz; Lai, Chun-Feng; Dankelman, Jenny; et al. (2021): Design of an affordable, modular implant device for soft tissue tension assessment and range of motion tracking during total hip arthroplasty. TechRxiv. Preprint.

https://doi.org/10.36227/techrxiv.16942879

$\mathrm{DOI}$

10.36227/techrxiv.16942879 


\title{
Design of an affordable, modular implant device for soft tissue tension assessment and range of motion tracking during total hip arthroplasty
}

\author{
Jonathan CJ Wei ${ }^{1}$, Bryan Blaauw ${ }^{2}$, Dieter GM van der Pol ${ }^{1}$, Mauricio Cruz Saldívar ${ }^{1}$, Chung-Feng Lai ${ }^{1}$, Jenny \\ Dankelman ${ }^{1}$, Tim Horeman ${ }^{1}$ \\ ${ }^{1}$ Department of BioMechanical Engineering, Faculty of Mechanical, Materials and Maritime Engineering, Delft \\ University of Technology, 2628 CD Delft, The Netherlands \\ ${ }^{2}$ Department of Orthopaedics, Elkerliek Hospital, 5707 HA Helmond, The Netherlands
}

\begin{abstract}
Inexperienced surgeons undertaking hip arthroplasties are twice as likely to experience errors than their experienced colleagues, leading to dislocations, pain and discomfort for the patients. To address this issue, a new 3DOF force measurement system was developed and integrated in multiple new prototypes able to measure forces and movements intraoperatively in 3D. The prototypes were evaluated in three cadaver trials, with the goal of providing surgeons objective data to help determine the optimal implant fit and configuration. The devices comprise deformable polymer material that provide strain-based displacements measured with electromagnetic-based sensors and inertial measurement unit (IMU) for motion data. Device results show an accuracy of approx. $2 \mathrm{~N}$ and a sensitivity of approx. $1 \mathrm{~N}$. Cadaver results indicated that soft tissue forces on the hip joint peak in the order of $\sim 100 \mathrm{~N}$ and trend with positions of the leg during range of motion (ROM) tests, although force patterns differ between each cadaver. We propose that by monitoring forces and force patterns, in combination with standardised ROM tests, anomalies could be detected and corrected during surgery.
\end{abstract}

Keywords: Medical device, surgery, hip arthroplasty, soft tissue balance, hip stability

\section{INTRODUCTION}

Hip replacement is a surgical procedure undertaken to relieve osteoarthritis pain $(\sim 63 \%)$ [1] and to treat fractures by replacing the hip joint with an implant, commonly consisting of a shell, liner, head and broach (stem), as shown in Figure 1 (a-b). In the case of a total hip replacement (THA), both the femoral head and acetabulum are replaced with artificial ball and socket joint. It is in fact the most common arthroplasty procedure in The Netherlands, with more than 40.000 operations carried out in 2019 and steadily increasing [2]. Also, an increasing number of younger population will be receiving this treatment [3], [4] while demanding more from the treatment to suit their more active lifestyles at the same time. THA prevalence between gender is approximately $55 \%-45 \%$ split between women and men [4], and thus a high degree of personalised treatment is necessary due to body geometry differences. Therefore, improvements to implant fit accuracy and soft tissue balancing around the hip joint are necessary. One method to achieve this is through force measurements during the fitting of the implant to sense the tensioning of the surrounding soft tissues, which can be adjusted by changing the implant geometries during the surgery. A more well-balanced hip can lead to a quicker recovery to normal daily activities.

Soft tissue balance of THA is less investigated than that of total knee arthroplasties (TKAs) [5], [6], even though the outcomes of THAs also rely on the surgeon's experience to determine properly balanced soft tissues. Instability is the biggest cause of early implant failure, typically within five years of surgery [7]. Conventionally, a conservative approach would be to provide sufficient tension through slightly higher offsets to ensure stability, although this does come with drawbacks such as pain, fatigue and longer recovery time until the muscles adapt over time. Larger femoral heads provide more stability and higher range of motion (ROM), but at the expense of higher impingement risks, liner fracture (due to thinner material) and higher wear rate because of the larger moment arm [8]. Too little tension, on the other hand, risks limping and even dislocation if the offset is not sufficiently restored [9]. Arguably, the optimum range of a "balanced" soft tissue tension within the hip is not simple to determine and is also subjective amongst the surgical professionals.

The implant configuration is first predetermined using $\mathrm{x}$-ray imaging and then confirmed intraoperatively with trial components before selecting a permanent implant by 
manipulating the leg in a series of movements with a ROM test, while the surgeon feels the muscles' resistance during the movements. The problem with the current ROM tests is that they are subjective, and there is no standard test protocol. Tang et al. [10], designed an inertial measurement unit (IMU) sensor-based implant and tested the accuracy/drift in vitro. Several studies indicate that soft tissue tension is related to pain (for instance in the groin and knee), dislocation, range of motion of the hip and limping [11] [13]. Furthermore, the most common errors in THAs are made by inexperienced surgeons [7], [14], with up to twice as many dislocation incidences than experienced surgeons, decreasing by $\sim 50 \%$ for every ten procedures performed annually, and plateauing after approximately 30 operations [15]. (a)
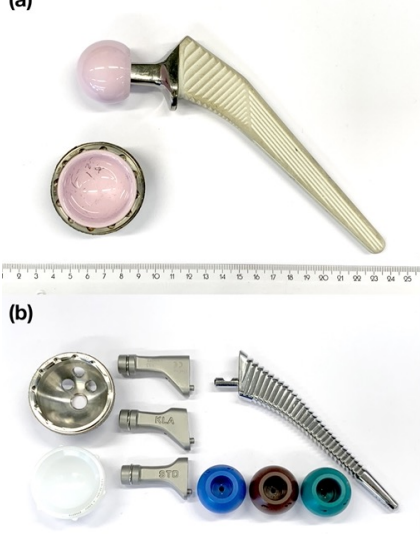

(c)

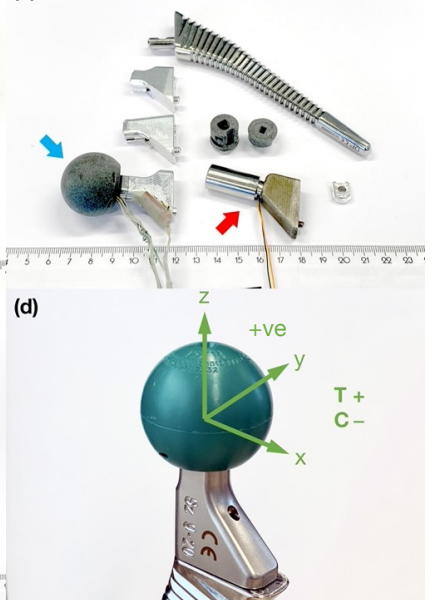

(e)

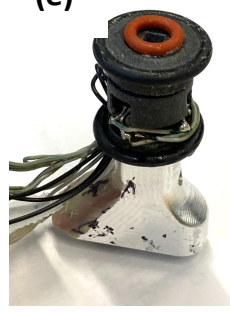

Figure 1. The stock implant (Corail Hip Systems, DePuy Synthes, Johnson\&Johnson, Warsaw IN, USA) used as the starting basis for the design of the instrumented prototypes. (a) The permanent implant and (b) the temporary (trial reduction) implant with various modular neck and head geometries. (c) The instrumented prototype with instrumented necks and a modified head. Blue arrow indicating 3DOF prototype and red arrow indicating $1 \mathrm{DOF}$ prototype. (d) The coordinate system used in this report; tension (or compression from the negative side of the axis) is positive while compression is reported as negative. (e) The inside of the 3DOF implant with the head removed showing the radial and axial O-rings, sensor placement and wiring.

Researchers of the Orthoload implants developed three generations instrumented "Hip I-III" of one-off versions of permanent hip implants that were used in clinical validation studies [16]-[18]. These latter implants were able to measure hip joint forces in everyday movement activities using embedded sensors that are powered through electromagnetic induction. While their work may initially appear to be similar to ours, our ultimate goal and design approach are fundamentally differenwt. The goal of this study is to measure and fine-tune the implant selection for hip replacements during the procedure. It is also therefore not about quantifying the forces experienced in the hip after the procedure. Although we expect forces experienced in everyday activities to be different from our scope - lying down, under anaesthesia, the work from Orthoload provides a useful point of comparative reference.

We propose a measuring system for surgeons to obtain an objective force reference in combination with the standardised ROM tests. The system was developed based on the design of a commercially available trial implant (DePuy Synthes Corail) (Figure 1 (b)) to remain simple, modular, and affordable, while keeping the technical requirements including biocompatibility, water resistance, sterilisation compatibility, appropriate sizing, sensing magnitude, accuracy, safety of use, reliability and user friendliness in mind. The bespoke neck and head components work with existing trial implants and the testing procedure regarding ROM trials. We first conceived a $1 \mathrm{DOF}$ device in the axial direction of the neck of the hip implant, as a proof of concept, since we expect most of the forces are transmitted in that direction. Then, the sensing capability was increased to 3DOF by addition of an IMU for motion sensing. The prototypes were tested and calibrated with a benchtop universal testing machine, and two pilot cadaver studies ( $\mathrm{n}=3$ in total) were carried out to evaluate the prototypes.

\section{MATERIALS AND METHODS}

\subsection{Design rationale}

A temporary trial reduction implant based on a commercially available design has been made. The design can be swapped out easily during the intraoperative fitting process. The prototype uses linear Hall-effect sensors in combination with a compliant material to measure small deformations $(\sim 1 \mathrm{~mm}$ at $30 \mathrm{~kg}$ load) within the temporary implant head. Hall-effect sensors were chosen because of their simplicity and because they were effectively used in a knee balancer unit before [5]. These Hall-sensors use a magnetic flux to determine their relative position to the source. Compliance of the structure was provided by a rubber O-ring between a neodymium magnet and the Hall-

secure the head in its position and to prevent. All components were selected to meet autoclave sterilisation (temperature, pressure, humidity) requirements; low-cost with the option to be reusable or single use if desired. Prototypes are wired and connected to a microcontroller (Arduino UNO, Arduino AG, Turin, Italy) and to a computer running Matlab (R2019a-R2020a Mathworks, Natick MA, USA).

\subsubsection{Implant design}

In the first phase of development, we conceived a 1DOF device in the axial direction, as a working principle, since we expect most of the forces are transmitted in that direction. The instrumented implant is designed based on the trial reduction broach of the Corail Total Hip System (DePuy 
Synthes, Johnson \& Johnson, Warsaw IN, USA) (Figure 1 (b)). Only the neck and head segments of the trial reduction components were utilised, while retaining their exterior geometry (Figure 1 (c)). Head size used was $\varnothing 32 \mathrm{~mm}$ with $+1 \mathrm{~mm}$ offset (as the standard) and neck geometry was the $125^{\circ}$ High Offset Coxa Vara Collared (KLA) variant. For the $3 \mathrm{DOF}$ prototype, additional necks were designed having variable offset (with spacers): $135^{\circ}$ Standard offset (STD135 ) and $135^{\circ}$ High Offset (KHO) variants. Only the standard offset was used in the cadaver study, as this is the most commonly used option.

\subsubsection{Sterilisation compatibility}

Electronic components were tested in an autoclave (MELAtronic 23 EN, MELAG, Berlin, Germany) through a standard cycle typical of hospital sterilisation equipment (steam at $134^{\circ} \mathrm{C}, 2$ bar, 20 minutes) as per van der Pol, 2017 [19] to verify the survival of the components.

\subsubsection{First iteration: 1DOF prototype}

Device design: a $1 \mathrm{DOF}$ device was developed with milled and 3D printed stainless steel components. A hall sensor (A1302/A1308, Allegro MicroSystems, Manchester NH, USA) was implemented at the axial (z-axis) direction of the neck (see Figure 1 (d)). A magnet (5x1 mm circular N38) is attached to the opposite end and can slide freely (assuming small friction). Sandwiched in between is an O-ring (10x6x2 $\mathrm{mm}$ circular cross section VMQ silicone rubber) acting as the spring element.

Force-voltage calibration of 1DOF: a compression test on the head was carried out to determine the relationship between applied force and voltage output of the sensors with a universal testing machine from $0-0.85 \mathrm{~mm}$ at $0.05 \mathrm{~mm}$ step size with $5 \mathrm{~s}$ of rest. From this we obtain the approximate Hooke's law correlation between the amount of exerted force, compression of the O-ring, the distance between the magnet and the sensor and thus the sensor voltage reading. We also assume small hysteresis is experienced during the (de)compression of the O-ring. In other words, the energy lost between compressing and decompressing the O-ring is small enough to assume the same force-voltage relationship.

\subsubsection{Second iteration: 3DOF prototype}

Device design: a 3DOF iteration was developed based on the 1DOF prototype with sensing in 3 axes and added an inertial measurement unit (IMU) gyroscopic motion sensor. The axial Hall sensor and O-ring setup was retained but three more Hall sensors were added on the sides of the neck to measure in the x-y plane (see Figure 1 (d)). The inner neck component was 3D printed using glass filled nylon (PA 6 GF30, Hubs, Amsterdam, The Netherlands) while the neck base was made of CNC machined aluminium (7075-T651 grade aluminium, Weerg, Gardigiano, Italy). Magnets were N50 instead of N38 as used in the 1DOF version. Two larger O-rings $(15 \times 10 \times 2.5 \mathrm{~mm}$ circular cross section NBR nitrile rubber) on the neck, spaced equidistance apart from the hemisphere of the head provide displacement in the radial direction (Figure 1 (e)). We assume small displacements of the O-rings and that any deviation/pivot about its centre causing the magnetic field and the sensor to misalign is small. Some crosstalk effects due to the close proximity of different sensor-magnet pairs were isolated for during the processing of the data by measuring how much the forces change in all principal directions (when compressing in one principal direction) and subtracting that deviation during data processing (see Supplementary Figure 1). The $x-y$ plane Hall sensors were arranged $120^{\circ}$ apart as shown in Figure 2. To convert the readings to principal forces by considering the voltage change, the location, angle and the plane of the sensor when a force is applied (thereby changing the distance between the magnets and the sensors), the following equations were used:

$$
\begin{aligned}
& V_{x}=\frac{\sqrt{3}}{4}\left(V_{3}-V_{2}\right) \\
& V_{y}=\frac{V_{1}}{2}-\frac{V_{2}+V_{3}}{8} \\
& V_{z}=V_{4}
\end{aligned}
$$

Equation 3

Full derivation can be found in Supplementary information section 1.

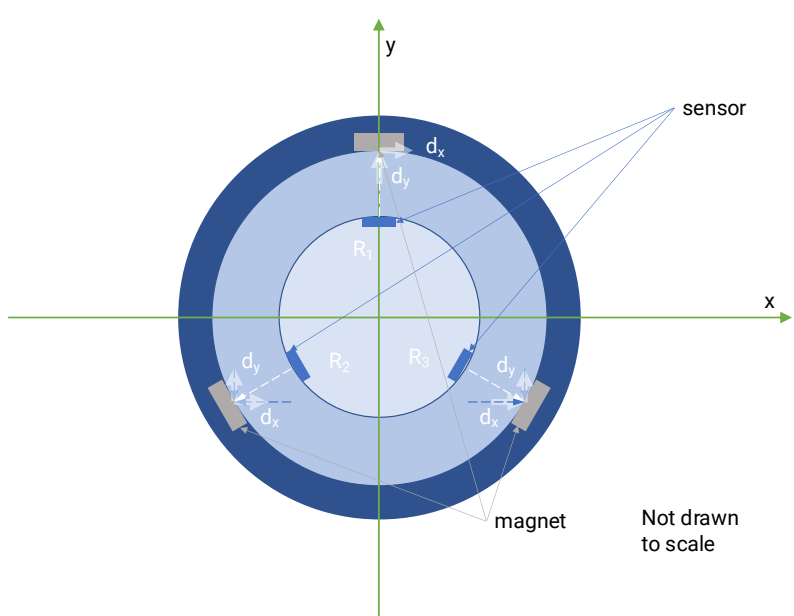

Figure 2. Schematic showing the $x-y$ plane cross section of the head with the three Hall effect sensors and their resolved force vectors.

Force-voltage calibration of 3DOF: a compression test with the 3DOF prototype was carried out on the universal testing machine (LR 5K, Lloyd Instruments, Bognor Regis, United Kingdom) under a linear load-unload profile at $1 \mathrm{~mm}$ $\mathrm{min}^{-1}$ to $1 \mathrm{~mm}$ displacement on 3 principal axes. The peak voltage reading is correlated with the peak force reading from the indenter. The force-displacement curves resemble a linear fit, therefore, a linear scaling factor was used. The right-hand rule convention for positive direction was applied such that compression is negative. The forces reported in the 3DOF data were zeroed in the neutral position, i.e. the relative forces are presented. To test the accuracy and sensitivity of the 3DOF sensor, it was additionally validated by hand with weight pieces of $2 \mathrm{~kg}+1 \mathrm{~kg}$ additionally, at three random angles.

Isolation of forces in the principal directions: interference and cross-talk from surrounding magnets may occur when the magnet positions change due to the displacement of the 
O-rings. The amount of interference is proportional to the amount of compression. The method to minimise the interference in one principal axis, the ratio between that and the cross talk from the two perpendicular axes are subtracted (for instance, the x-axis formula is shown in Equation 9). The overall result for this isolation method is shown in Supplementary Figure 1 and it appears that unwanted interference was minimised. Furthermore, the raw data is shown additionally with Matlab smoothing filters of Matlab functions medfilt1 and sgolayfilt. This was not used during the cadaver experiments.

$x_{n e w}=x-\frac{y^{2}}{x}-\frac{z^{2}}{x}$

Equation 4

Hysteresis and stress relaxation: the degrees of hysteresis and stress relaxation of the compliant material - the polymer O-ring material were examined. Both the top and side Orings underwent a linear load-unload, load-controlled compression using the universal testing machine at $0.67 \mathrm{~mm} / \mathrm{s}$ and $1 \mathrm{~mm} / \mathrm{s}$ to $95 \mathrm{~N}$ (load cell limit of $100 \mathrm{~N}$ ). For stress relaxation, a hold section of $30 \mathrm{~s}$ is added to the abovementioned test method between the loading and the unloading profiles. The relaxation curves were normalised for the two term Prony series function in Supplementary Figure 8. The Prony coefficient for the instantaneous modulus was obtained in the same method as Crichton et al. [20] (see for derivation). The equation used for the two-term Prony series is as follows:

$G(t)=1-g_{1}\left(1-e^{-\frac{t}{\tau_{1}}}\right)-g_{2}\left(1-e^{-\frac{t}{\tau_{2}}}\right) \quad$ Equation 5

Inertial measurement unit (IMU): a 9DOF IMU stick with the LSM9DS1 chip (SEN-13944, Sparkfun, Niwot CO, USA) was seated on the neck of the 3DOF unit to provide spatial coordinates to the user. The IMU connected to the same Arduino is used to process the Hall-effect sensors' information using Matlab functions ' $1 \mathrm{sm} 9 \mathrm{ds} 1$ ', 'readAcceleration', 'readAngularVelocity' and 'readMagneticField; complementaryFilter' and converted to rotation in the principal axes [21]. Because of the default output of the IMU and the angle it is mounted on the neck, the principal axes were calculated, such that the $\mathrm{x}$-axis points to the implant's shoulder, and the z-axis points to the head (see Figure 1 (d)). The recorded ROMs were reviewed in Matlab for verification using the accelerometer-gyroscopemagnetometer fusion function 'ahrsfilter'.

\subsection{Cadaver study}

\subsubsection{Approval statement}

The cadaver studies were approved by and carried out in accordance with the Tissue Bank guidelines of Erasmus Medical Centre, Rotterdam, The Netherlands.

\subsubsection{Test protocol}

The right legs of fresh (non-frozen-and-thawed/nonpreserved) cadavers $(n=3$, elderly age, exact age not provided) were used, having an already-exposed hip region from an earlier hip arthroplasty skills lab session using the anterior approach and bored femur and reamed acetabulum on the day. The rigor mortis phase past the initial time of death was avoided. One cadaver was used for the 1DOF trial while two others were used for the 3DOF trial separately. Originally a fourth cadaver was prepared. However, the tissue conditions were unsuitable for trials and thus the fourth cadaver was excluded from the study.

\subsubsection{Range of motion (ROM) protocol}

The cadaver was in supine position with the hip exposed through the anterior approach. The following tests were conducted for the 1 DOF implant trial $\left(0^{\circ}\right.$ means neutral position). Each movement was repeated 2-3 times.

1. External rotation from $\sim 0^{\circ}$ to $45^{\circ}$ then to $90^{\circ}$

2. Internal rotation from $\sim 0^{\circ}$ to $45^{\circ}$ then to $90^{\circ}$

3. Abduction from $\sim 0^{\circ}$ to $25^{\circ}$

4. Adduction from $\sim 0^{\circ}$ to $25^{\circ}$

5. Flexion of hip and knee from $0^{\circ}$ to $45^{\circ}$ then to $90^{\circ}$

6. Adduction-abduction to $\sim 25^{\circ}$ combined with a $45^{\circ}$ rotation

For the 3DOF implant trial, the following tests were conducted:

1. Push, pull (proximal-distal)

2. External rotation

3. Flexion, extension

4. Flexion, external (+internal*) rotation, extension

5. External rotation (with dislocation)*

*For the second cadaver trial only

We allowed a three second waiting time and zeroed the readings before the commencement of each test.

\section{RESULTS}

To design a soft tissue tension measuring device for hip arthroplasty, we first developed a 1DOF prototype using simple, off-the-shelf Hall-sensor based electronics and tested it in a cadaver study. This was followed up by an improved 3 DOF prototype which was tested in two cadavers.

\subsection{Device performance characteristics}

\subsubsection{Principal axes calibration}

The indentation example shown in Supplementary Figure 1 shows the raw readings (top row) converted to principal axes (middle row), and then with crosstalk effects minimised (bottom row). Both the crosstalk effects and the noise appeared to be filtered out well.

3.1.2 Rubber O-ring characteristics: hysteresis, stress relaxation and sterilisation compatibility

In linear load-unload indentations of two speeds $(0.67 \mathrm{~mm} / \mathrm{s}$ and $1 \mathrm{~mm} / \mathrm{s}$ ), most curves (Supplementary Figure 8) appeared to have minimal hysteresis effects for the O-ring material used in the device (the return curve follows closely 
with the initial curve; the area between the outbound and inbound curves are small).

In terms of stress relaxation, the load-hold-unload indentations of $30 \mathrm{~s}$ showed that the material's resistance to a constant force decreased sharply within 2-3 seconds after applying a constant force, and that after a fitting to a two term Prony series, we arrived at an average Prony series coefficient for each indentation velocity/O-ring type combination of 0.92-9.96 (Error! Reference source not $f$ ound.). In other words, the forces measured may be up to approximately $8 \%$ lower than the true force in the load-holdunload experiment after about $10 \mathrm{~s}$. Consequently, we take the stress relaxation effects are small as a reasonable approximation.

\subsection{Cadaver study}

\subsubsection{Insertion of the implant onto the stem in the cadaver}

The instrumented device was placed directly atop the stem in the same way as placing a regular neck and stem without hinderance to usability and user-friendliness. The data cable connected to the implant leads out the opening of the hip area to the Arduino and computer for recording and processing.

\subsubsection{IDOF prototype}

We observed distinct changes in force patterns of soft tissue forces acting on the implant head as the leg was moved during the range of motion trials. Figure 3 (a-f) shows, a force range of approximately 20-120 N, depending on the ROM. Distinct patterns were observed for specific positions, for instance, $(\mathbf{a}-\mathbf{b})$ at the peak $\left(90^{\circ}\right)$ of each internal/external rotation. Notably, at the halfway point of the motion $\left(45^{\circ}\right)$, the force reading was not exactly halfway between the force values corresponding to $0^{\circ}$ and $90^{\circ}$ and was closer to the force value of the neutral $\left(0^{\circ}\right)$ position than the $90^{\circ}$ position. For internal rotation (b), the midway force varied depending on the repetition, and an overall trend with decreasing in absolute force was observed. Both abduction and adduction (c-d) movements from the neutral position to $\sim 25^{\circ}$ produced a peak force in principal axes of approximately $120 \mathrm{~N}$ and $100 \mathrm{~N}$, respectively. For the flexion of the hip in combination of the flexion of the knee (e) from $0^{\circ}$ to $45^{\circ}$, the forces were higher than at $90^{\circ}$. In the final combined ROM (f), the forces peaked at the end positions $\left(25^{\circ}\right)$ of abduction and adduction of approximately $100 \mathrm{~N}$, and a small dip to approximately 80 $\mathrm{N}$ was observed when the leg was moving from abduction to adduction. The additional exorotation movement appeared to differ between the first and second cycle - the first force reading of the first exorotation was closer to the starting force than the second exorotation. During certain movements such as an internal rotation (b), the peak forces deceased after each successive motion, although the pattern generally remained the same. In most cases, the start and finish force readings at the neutral position differ (a-b at $\sim 40 \mathrm{Nm}$ c-f at $\sim 25 \mathrm{~N}$ ), with the exception of the (d) adduction ROM.
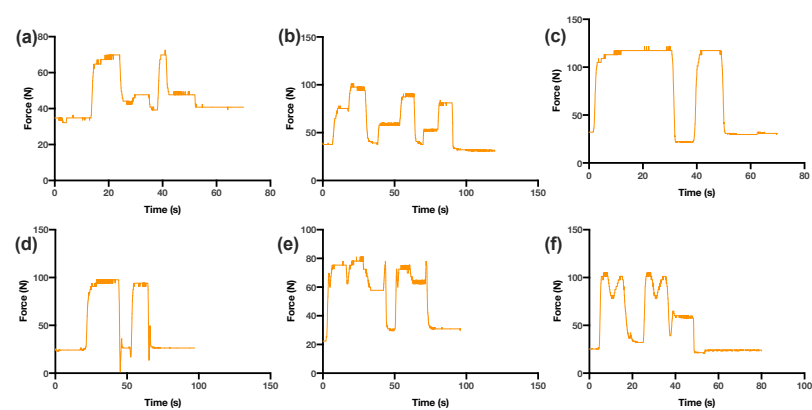

Figure 3. 1DOF in the axial direction showing various ranges of motion performed on one cadaver: (a) external rotation of the hip, (b) internal rotation, (c) abduction, (d) adduction, (e) Flexion and (f) abduction and adduction, plus external rotation.

\subsubsection{DOF prototype}

The results of the cadaver study carried out with the 3DOF prototype ( $2 \mathrm{x}$ replicates) are shown in left and right columns of Figure 4 - Figure 7. The forces (top row) are in a range of approximately $-40 \mathrm{~N}$ to $100 \mathrm{~N}$ for the cadaver $\mathrm{C} 1$ and within $-70 \mathrm{~N}$ to $50 \mathrm{~N}$ for cadaver $\mathrm{C} 2$. Additional angular velocity readings (bottom row) are presented, illustrating the 'consistency' of the surgeon's input and speed in moving the leg through the various ROMs, which peaked at approximately $60 \mathrm{rad} / \mathrm{s}$ for most movements. The additional validation done by hand with weight pieces, shown in Supplementary Figure 9, shows an estimated accuracy of approx. $2 \mathrm{~N}$ and a sensitivity/resolution (taken as the coarsest fluctuation before Matlab filter smoothing) of approx. 0.85$1 \mathrm{~N}$. Raw readings directly from the sensors are shown in Supplementary Figure 5.

During push-pull: the first movement (Figure 4), most of the compression force in the head is in the $\mathrm{z}$ axis direction. For both replicates, the peak force for each compression is at approximately $40 \mathrm{~N}$ and $70 \mathrm{~N}$. Note that the final position does not necessarily return to zero. The initial "moving the leg into position" in the second replicate showed a much higher (almost $40 \mathrm{rad} / \mathrm{s}$ ) rotation rate than the push-pull motion, and as expected, the angular velocity for the entire ROM remains small $(>10 \mathrm{rad} / \mathrm{s})$ as the leg does not rotate (see Supplementary Figure 3 C1-C2 push-pull).
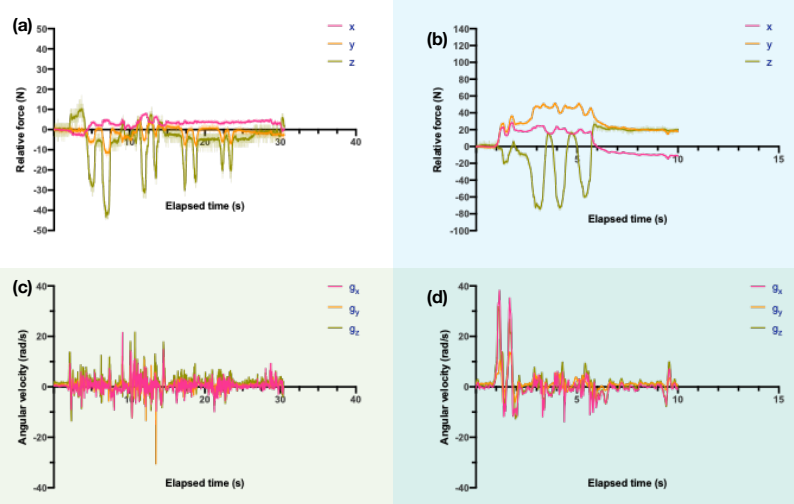

Figure 4. 3DOF of the push-pull (proximal-distal) range of motion performed on two cadavers (left and right columns respectively), with the top row $(\mathrm{a}, \mathrm{b})$ showing the net resultant force in each principal direction and the bottom row showing the angular velocities (c, d) from the IMU showing the rotational movements of the hip. 
During exorotation (Figure 5), the two cadavers exhibited distinct patterns during the ROM albeit different magnitudes between the two replicates. The forces all peaked at the end (limit) of the movement corresponding to where the angular velocity curves cross the $\mathrm{x}$ axis. The principal $\mathrm{y}$ axis direction reading of the end section of the ROM for the first replicate appeared to have plateaued at approximately $50 \mathrm{~N}$ instead of close to zero.
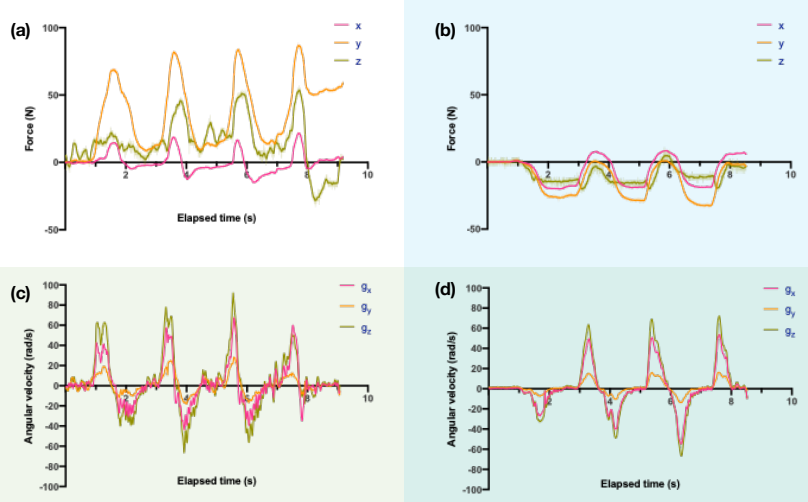

Figure 5. 3DOF measurements of the hip external rotation ROM performed on Cadaver 1 (left) and Cadaver 2 (right), with the top row $(\mathrm{a}, \mathrm{b})$ the net resultant force in each principal direction and the bottom row the angular velocities (c, d).

Forces observed for the flexion movement in Figure 6 show, again, two different magnitudes acting on the joint, although the patterns were still discernible especially in Cadaver $\mathrm{C} 1$. A tightening of soft tissues was observed at the peaks of the flexion movement, shown primarily with a positive $\mathrm{z}$ axis reading. The y axis reading of Cadaver $\mathrm{C} 2$ shows a sudden decrease during the second cycle, but this is not reflected in the IMU data. Forces for Replicate 2 also appeared to decrease first than increase at the peak of the movement, likely coinciding with the subtle movement endorotation between 1-2 second timeframe. The angular velocity patterns show that the movements of the ROMs between the two were comparable, although for Cadaver $\mathrm{C} 1$, the rotation rate was smaller than Cadaver C2, likely due to large resistance coming from soft tissues.
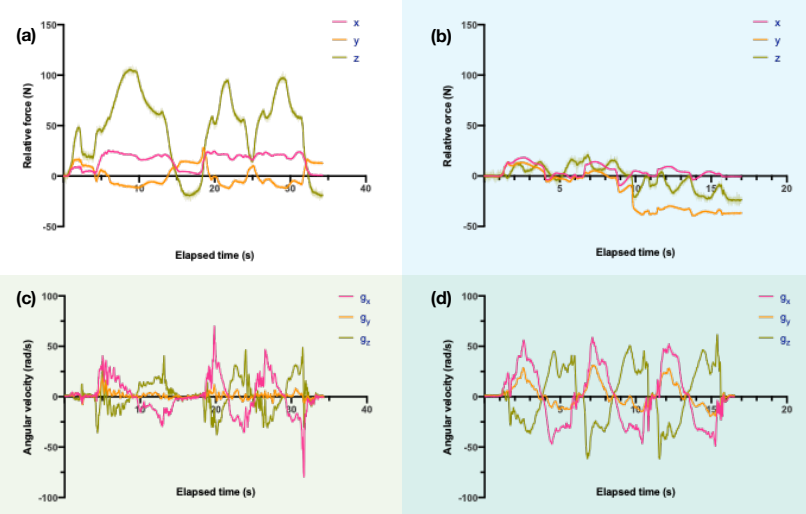

Figure 6. 3DOF measurements of flexion-extension ROM performed on Cadaver 1 (left) and Cadaver 2 (right), with the top row (a, b) the net resultant force in each principal direction and the bottom row the angular velocities (c, d).
In the combined ROM of flexion, external rotation and extension, the data is presented in Figure 7. Consistently, Cadaver $\mathrm{C} 1$ exhibited higher forces (peaks at approximately $-70 \mathrm{~N}$ to $100 \mathrm{~N}$ ) whereas Cadaver 2 showed much smaller magnitudes at less than $-20 \mathrm{~N}$ to $20 \mathrm{~N}$. Cadaver 1's force readings and patterns were comparable to the flexion ROM as presented in Figure 6, but with the additional dip accounting for the external rotation. Cadaver $\mathrm{C} 2$, however, also displayed distinct patterns, although while the forces were much smaller, the external and internal movements were captured in the forces as a dip in the $\mathrm{x}$ and $\mathrm{y}$ axes and then an increase in the same axes. Instead of returning to the neutral position after an external rotation of Cadaver $\mathrm{C} 2$, the motion was continued to an internal rotation.
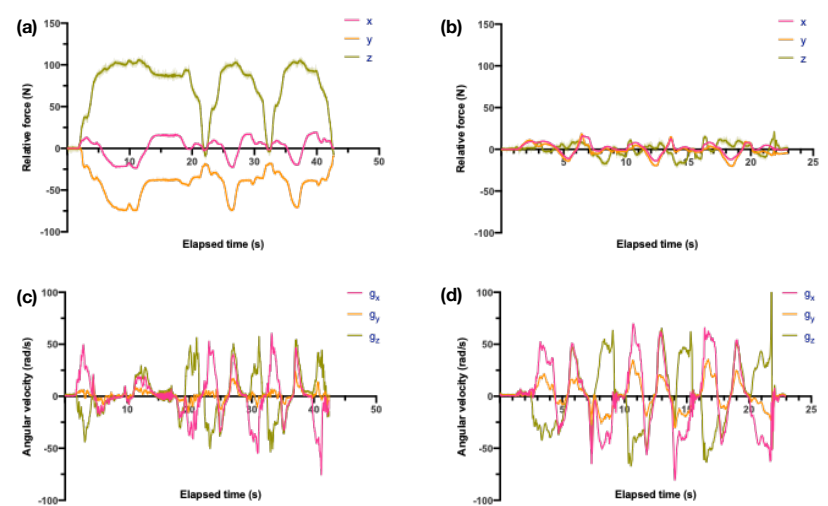

Figure 7. 3DOF measurement of the hip flexion, external rotation and extension ROM performed on Cadaver $\mathrm{C} 1$ (left), and an additional, internal rotation on Cadaver 2(right) with the top row $(\mathrm{a}, \mathrm{b})$ the net resultant force in each principal direction and the bottom row the angular velocities (c, d)

In the final ROM carried out additionally at the end of the experiment session, a combined external rotation with deliberate dislocation motion was carried out as an exploratory test following the final ROM mentioned above of Cadaver C2, and the result is presented in Supplementary Figure 6. The force curves began similar to the exorotation ROM of Figure 5 (c). However, a deliberate manoeuvre of the hip joint to cause it to dislocate was identified as a jolt (just before $10 \mathrm{~s}$ elapsed time) in the figure.

Acceleration and magnetic field data from the IMU: the acceleration magnitudes are shown in Supplementary Figure 3 and reported in standard gravity $g\left(9.81 \mathrm{~m} / \mathrm{s}^{2}\right)$. From here we observed that the y axis is at approximately 1 when at neutral position from gravity. The readings of all three axes illustrate the position of the leg during the range of motion with the relation between the implant reference frame (Figure 1 (d)) and the earth reference frame. The magnetometer data presented in Supplementary Figure 4) with units in gauss, also showing some similarities, when compared with the acceleration data in terms of rise and fall of patterns in the principal axes, is used to provide orientation reference when the motion is reanimated later in Matlab.

\section{DisCUSSION}

In this paper, we presented two design iterations of a novel, low-cost medical device prototype, capable of measuring 
forces and orientation data for use during hip arthroplasties and trialled them on three cadavers. The ability to obtain soft tissue tension measurements of the hip easily will especially assist inexperienced surgeons, who cannot 'feel' the right level of tension, as well as experienced surgeons to provide patients with adequate hip stability. To our knowledge, this is the first device designed to measure soft tissue tension of the hip intraoperatively, tested in an in situ pilot study.

Overall, the force profiles of ROMs carried out on 3 cadavers (1x 1DOF, 2x 3DOF with motion data) exhibited different magnitudes, with peaks up to approximately $120 \mathrm{~N}, 100 \mathrm{~N}$ and $50 \mathrm{~N}$ for Cadavers $1, \mathrm{C} 1$ and $\mathrm{C} 2$, respectively, with motion profiles showing the position at which these peaks happen - typically at the extreme ends of the motion. The rotational rates of the hip manipulated by the surgeon during the test also appeared more consistent (peaking at approximately $50 \mathrm{rad} / \mathrm{s}$ ) in the 3DOF study than the forces measured. The device was inserted into the cadavers' hips without difficulty or change to standard surgical protocol apart from the additional cable that connects from the device to the Arduino. In our case, the surgeon continues with the operation and the ROM tests, while another OR team member monitors the data readout.

The forces are relative to the neutral position at the beginning of the ROM. In many cases, the forces at the end of the ROM recording do not return to zero. We suspect this could be due to some bodily fluids have shifted to other regions, and/or the fact that soft tissues are highly viscoelastic and possess hysteresis properties, so residual forces remain acting on the joint and sufficient time ( $\sim 1$ minute) and 'loosening' (i.e. a few 'wiggles') of the leg back at its neutral position should be carried out. Furthermore, the overall decreasing trend in absolute forces is probably due to the stretching and relaxing of the cadaver muscles. Additionally, soft tissue behaviour of a cadaver may be different than that in a living patient. Regardless, in future, device accuracy could be calibrated or checked prior and after an experiment to identify drifts.

Interestingly, even though the force readings appear to differ between the two 3DOF cadavers, the angular rotational rates, as well as the acceleration vectors suggest that the ROMs were carried out consistently between the two. This discrepancy and the residual forces, most likely down to individual differences between the two cadavers (which further stresses the need for a higher level of personalised treatments in orthopaedic surgery with tissue stability and balancing due to the variability surgeons need to recognise), could eventually be used as an indicator for surgeons to monitor for unexpected forces or force patterns at each position of the ROM tests, taking into account the body geometries and soft tissue volumes. We also expect different approaches (e.g. posterior, lateral and anterior) to have a significant influence on the overall force measurements of the soft tissues.

We propose that this device eventually can be used to ensure ROMs are carried out consistently (through the use of IMU movement data) - e.g. have the surgeon check the flexion or rotation angle and the rate of motion is carried out to a predetermined position and velocity, and check if the resistance of the soft tissue tension is as expected with the force readings. With sufficient repetitions, experienced surgeons can begin devising "gold standard" guidelines on ROM protocols - at the moment, this differs per surgeon. The following step would be to use the collected (in vivo and in situ and identify differences) patient body build data (bone geometry and soft tissue volume), measured forces and movements (and through the input from multiple surgeons to identify 'good' and 'bad' cases) to build a predictive model. We propose to normalise the forces and use the force ratios at different ROM positions as a point of reference for modelling. We foresee this technology will eventually supplement current surgeries by suggesting to the surgeon intraoperatively, for a given patient and a given ROM, the ideal range of force response. If the measured force is outside the "optimum" range, then the device could prompt the surgeon to adjust the implant or to change the geometry, such as neck angle and/or offset, implant and/or head/cup sizes. This will ultimately lead to a more personalised medical approach, tailoring each implant operation more specifically to suit individual patients. At the same time, we also minimise the subjectivity of different surgeons.

Furthermore, data collected from in situ conditions in our study may be different compared to in vivo conditions - we expect somewhat higher force readings during in vivo readings, at least partly due to due to circulation of bodily fluids [22], but it is still useful as an insight and a steppingstone before the prototype is tested in clinical settings. Nevertheless, we were able to establish the order of magnitude of typical forces $(\sim 100 \mathrm{~N})$ experienced for in situ conditions and can optimise subsequent device designs to provide a higher resolution for this expected range of forces. Additionally, the values we obtained comparing to published data from e.g. Orthoload were in the same order of magnitude - granted that their values were taken from living patients without anaesthesia. They reported a peak load of approximately $+150-250 \mathrm{~N}$ for $45^{\circ}$ flexion and $\pm 150 \mathrm{~N}$ for hip abduction (Orthoload online database) [18]. So, a further study may be interesting to compare differences between in vivo datasets with in situ cadaver experiments, as well as in vivo anaesthetised patient experiments. We hypothesise in the latter case, the muscles will be completely relaxed and may give lower force readings. On top of this, we were also able to distinguish that force readings correlate to positions of ROM movements. The ROM protocols we used in this study are also useful - at a minimum, surgeons should carry out a flexion-extension ROM, external and internal rotations, and a combination of those movements to capture as much of a range of the hip movement as possible.

Regarding the (in)accuracy of using polymer materials (rubber) as the deformable material within the implant, we believe after showing the materials have small hysteresis (e.g. $<10 \%$ ) and viscoelastic effects (at least for the tested compression rate and the linear load-unload tests anyway), it is acceptable for our purpose when measuring muscle tissues 
that have greater hyperelastic and hysteresis effects due to tissue fluids [23]. A proportional-integral-derivative (PID) loop built into the post-processing of the data may also mitigate this effect [24], although this may slow down the refresh/sampling rates of the overall system as well as introducing unnecessary complications to the device. Not every surgeon will perform the movements the same way, and would be difficult to implement with human factors involved.

Rubber O-rings used herein were also found unable to withstand autoclave sterilisation environment, so new, sterile O-rings must be used for future in vivo studies or alternatively, a different method of sterilisation. Alternative materials such as metallic-based deformable structures could eventually substitute the O-ring (would also eliminate any undesirable polymer characteristics such as hysteresis and stress relaxation), however, another material is required to seal the internal structure as the O-ring also doubles as a fluid barrier and may require custom manufacturing. Furthermore, it is plausible that increasing the accuracy marginally may substantially increase the cost and complexity of this device. Regardless, to minimise capturing hyperelastic and hysteresis effects from both the rubber O-ring and biological tissues during the experiments in the current design, we propose that by waiting three seconds to allow the rubber and soft tissues to enter steady state of relaxation [25], we minimise the variability from soft tissues. This should be an important step to include in all future in situ and in vivo studies.

For imminent future work, apart from those longer-term data-collection experiments already mentioned, an immediate improvement to the current device design can be further locking any potential rotation of the head with respect to the neck, as any rotation/unalignment will influence the $\mathrm{x}$ and $y$-axes readings when the distance between the Hall sensors and the magnets changes. Although not directly monitored in the experiments, we suggest changing the fitting/base of at least one of the O-rings to a triangular shape to improve the alignment of the magnet-sensor pairs. An example of this is shown in Figure 8.

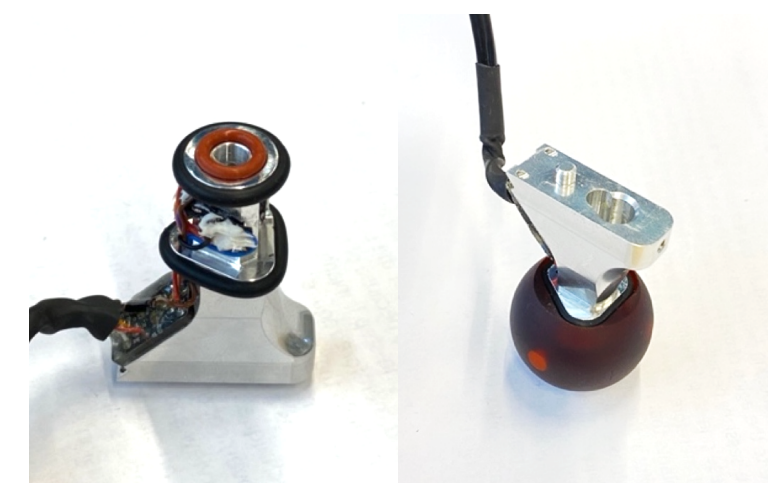

Figure 8. Improved triangle base design with head rotation lock allowing more precise sensor-magnet alignment for future use. (a) is without the head, viewed from the top/side, and (b) viewed from below, with the head fitted.

\section{CONCLUSION}

In this work, we developed two force measuring hip implant prototype devices, first in 1DOF as a proof-of-principle, then in 3DOF with integrated IMU capabilities, and used them in three cadaver ROM trials. The goal is to provide surgeons with real-time, objective measurements to facilitate them during surgery in selecting the optimal implant position and geometry for the patient to improve patient outcomes, such as minimising pain and the likelihood of dislocations from an unbalanced hip. Stress relaxation and hysteresis of the device structure was deemed to be small $(<10 \%)$ especially when compared to that of biological tissues. Thus, we proposed that the use of off-the-shelf materials was suitable to keep the design simple and low-cost. In the experiments, we found force values ranging in the order of $\sim 100 \mathrm{~N}$, also comparable to literature data, however, both force magnitudes and patterns differ between cadavers. We suggest that using both force and position data could help identify undetected anomalies in implant fit when ROM tests are carried out by the surgeon. Finally, we suggest that future work should focus on device refinement and optimisation for clinical use, investigate a baseline of "optimally balanced hip", devise standardised guidelines for ROM tests and to collect data in (pre)clinical trials, to build up a database of patient body geometry and force measurements. These investigations will eventually lead to a higher level of personalised treatment for individual patients, increased success rate and quality of THAs, while reducing the cost on the healthcare system (e.g. reduced recovery time) and added value to patients, healthcare providers and implant makers.

\section{DATA AVAILABILITY}

The datasets generated and/or analysed during the current study are available from the corresponding author on reasonable request.

\section{FUNDING}

This study was funded by the ZonMW IMDI Technology for Sustainable Healthcare - Sensing in Surgery \#67867.

\section{COMPETING INTERESTS}

The authors declare no competing interests. DePuy Synthes did not perform any of the experiments nor played a role that could have influenced the outcome of the results. DePuy Synthes provided demo trial implants and CAD drawings as a collaborative partner listed in this grant.

\section{AUTHOR CONTRIBUTIONS}

JCJW wrote the manuscript and was the main contributor to the 3DOF prototype development, data analysis and associated experiments. DvdP was the main contributor to the 1DOF prototype development, data analysis and associated experiments. BJB performed the ROMs and provided data analysis and critical oversight to the manuscript. MCS assisted with the design and mechanical testing of the materials. CFL assisted with validating the derivation of the $3 \mathrm{DOF}$ equations. TH and JD provided 
critical oversight to the prototype designs, data analysis and manuscript revisions.

\section{ACKNOWLEDGEMENTS}

We would like to thank the Erasmus MC and its staff for helping to organise and to allow us to perform the cadaver studies. We also thank DePuy Synthes for the support and to allow us to perform the experiments with their resources such as the cadaver studies. In addition, we thank Mohammed Mirzaali Mazandarani and Sander Leeflang for assisting with the initial setup and training of the LR 5K.

\section{REFERENCES}

[1]J. Wright, S. Rudicel, and A. Feinstein, 'Ask patients what they want. Evaluation of individual complaints before total hip replacement', J. Bone Joint Surg. Br., vol. 76-B, no. 2, pp. 229-234, Mar. 1994, doi: 10.1302/0301-620X.76B2.8113282.

[2]Dutch Arthroplasty Register (LROI), 'Online LROI annual report 2019', 2019. Accessed: Jun. 30, 2020. [Online]. Available: https://www.lroirapportage.nl/media/pdf/PDF\%20Online\%20LROI\%20annual\%20report\% 202019.pdf

[3]M. Sloan and N. Sheth, 'Changing Demographics in Primary and Revision Total Joint Arthroplasty, 2000-2014.', presented at the American Academy of Orthopaedic Surgeons 2018 Annual Meeting, New Orleans, Mar. 2018

[4]J. P. McAuley, E. S. Szuszczewicz, A. Young, and C. A. S. Engh, 'Total Hip Arthroplasty in Patients 50 Years and Younger.', Clin. Orthop. Relat. Res. 1976-2007, vol. 418, pp. 119-125, Jan. 2004.

[5]T. Paszicsnyek, 'Dynamic ligament balancing system (dlb)', WO2017185108A2, Nov. 02, 2017 Accessed: Jun. 23, 2021. [Online]. Available: https://patents.google.com/patent/WO2017185108A2/en

[6]K.-J. Cho, J.-K. Seon, W.-Y. Jang, C.-G. Park, and E.-K. Song, 'Objective quantification of ligament balancing using VERASENSE in measured resection and modified gap balance total knee arthroplasty', $B M C$ Musculoskelet. Disord., vol. 19, no. 1, p. 266, Jul. 2018, doi: 10.1186/s12891-018-2190-8.

[7] S. D. Ulrich et al., 'Total hip arthroplasties: What are the reasons for revision?’, Int. Orthop., vol. 32, no. 5, pp. 597-604, Oct. 2008, doi: 10.1007/s00264-007-0364-3.

[8]R. J. Tansey, G. L. Green, and F. S. Haddad, 'Large diameter heads: Is bigger always better?', Semin. Arthroplasty, vol. 26, no. 1, pp. 16-19, Mar. 2015, doi: 10.1053/j.sart.2015.04.004.

[9]R. Malhotra, Mastering Orthopedic Techniques: Total Hip Arthroplasty. JP Medical Ltd, 2011.

[10] H. Tang et al., 'Monitoring hip posture in total hip arthroplasty using an inertial measurement unit-based hip smart trial system: An in vitro validation experiment using a fixed pelvis model', J. Biomech., vol. 97, p. 109415, Dec. 2019, doi: 10.1016/j.jbiomech.2019.109415.

[11] R. B. Bourne and C. H. Rorabeck, 'Soft tissue balancing: The hip', $J$. Arthroplasty, vol. 17, no. 4, Supplement 1, pp. 17-22, Jun. 2002, doi: 10.1054/arth.2002.33263.

[12] D. Longjohn and L. D. Dorr, 'Soft tissue balance of the hip', $J$. Arthroplasty, vol. 13, no. 1, pp. 97-100, Jan. 1998, doi: 10.1016/S08835403(98)90082-1.

[13] P. Kosev, B. Valentinov, Y. Andonov, and C. Sokolov, 'SOFT TISSUE BALANCING IN TOTAL HIP ARTHROPLASTY', $J$. IMAB Annu. Proceeding Sci. Pap., vol. 21, no. 1, pp. 752-756, Mar. 2015, doi: 10.5272/jimab.2015211.752.

[14] J. S. Melvin, T. Karthikeyan, R. Cope, and T. K. Fehring, 'Early Failures in Total Hip Arthroplasty - A Changing Paradigm', $J$. Arthroplasty, vol. 29, no. 6, pp. 1285-1288, Jun. 2014, doi: 10.1016/j.arth.2013.12.024.

[15] U. Hedlundh, L. Ahnfelt, C. H. Hybbinette, J. Weckstrom, and H. Fredin, 'Surgical experience related to dislocations after total hip arthroplasty', J. Bone Joint Surg. Br., vol. 78, no. 2, pp. 206-209, Mar. 1996. [16] M. O. Heller, G. Bergmann, G. Deuretzbacher, L. Claes, N. P. Haas, and G. N. Duda, 'Influence of femoral anteversion on proximal femoral loading: measurement and simulation in four patients', Clin. Biomech., vol. 16, no. 8, pp. 644-649, Oct. 2001, doi: 10.1016/S0268-0033(01)00053-5.
[17] G. Bergmann, F. Graichen, and A. Rohlmann, 'Hip joint loading during walking and running, measured in two patients', J. Biomech., vol. 26, no. 8, pp. 969-990, Aug. 1993, doi: 10.1016/0021-9290(93)90058-M.

[18] G. Bergmann, A. Bender, J. Dymke, G. Duda, and P. Damm, 'Standardized Loads Acting in Hip Implants', PLOS ONE, vol. 11, no. 5, p. e0155612, May 2016, doi: 10.1371/journal.pone.0155612.

[19] Dieter van der Pol, 'Assessment of soft tissue tension during total hip arthroplasty: measuring the forces in the neck of a prosthesis', Delft University of Technology, Delft, 2017. [Online]. Available: https://repository.tudelft.nl/islandora/object/uuid\%3A7348e6ed-cabe-432fa1f2-3144238e 78 f2

[20] M. L. Crichton, B. C. Donose, X. Chen, A. P. Raphael, H. Huang, and M. A. F. Kendall, 'The viscoelastic, hyperelastic and scale dependent behaviour of freshly excised individual skin layers.', Biomaterials, vol. 32, no. 20, pp. 4670-4681, Jul. 2011, doi: 10.1016/j.biomaterials.2011.03.012. [21] 'Estimate Orientation Through Inertial Sensor Fusion - MATLAB \& Simulink - MathWorks Benelux'. https://nl.mathworks.com/help/fusion/examples/estimate-orientationthrough-inertial-sensor-fusion.html (accessed Jul. 07, 2020).

[22] J. C.-J. Wei, 'The key biomechanics of the skin for the translation of microscale medical devices from animal models to humans', The University of Queensland, Brisbane, 2018. Accessed: Jun. 23, 2021. [Online]. Available: https://espace.library.uq.edu.au/view/UQ:d0488b4

[23] J. C. J. Wei et al., 'Space- and time-resolved investigation on diffusion kinetics of human skin following macromolecule delivery by microneedle arrays', Sci. Rep., vol. 8, no. 1, p. 17759, Dec. 2018, doi: 10.1038/s41598-018-36009-8.

[24] B. Ding and Y. Li, 'Hysteresis Compensation and Sliding Mode Control with Perturbation Estimation for Piezoelectric Actuators', Micromachines, vol. 9, no. 5, p. 241, May 2018, doi: 10.3390/mi9050241. [25] J. C. J. Wei, G. A. Edwards, D. J. Martin, H. Huang, M. L. Crichton, and M. A. F. Kendall, 'Allometric scaling of skin thickness, elasticity, viscoelasticity to mass for micro-medical device translation: from mice, rats, rabbits, pigs to humans', Sci. Rep., vol. 7, no. 1, Art. no. 1, Nov. 2017, doi: 10.1038/s41598-017-15830-7. 